\title{
XXXIII. On measurements on the earth's surface perpendicular to the meridian
}

\author{
J. Ivory Esq. M.A. F.R.S.
}

To cite this article: J. Ivory Esq. M.A. F.R.S. (1828) XXXIII. On measurements on the earth's surface perpendicular to the meridian, Philosophical Magazine Series 2, 4:21, 189-194, DOI: $10.1080 / 14786442808674775$

To link to this article: http://dx.doi.org/10.1080/14786442808674775

曲 Published online: 10 Jul 2009.

Submit your article to this journal $₫$

Џlll Article views: 1

Q View related articles ๘ 
Mr. Ivory on Measurements of perpendicular Degrees. 189

The plummet $P$, and the screws at $L$, enable the operator to detect and rectify any deviation from perpendicularity in the instrument.

XXXIII. On Measurements on the Earth's Suiface perpendicular to the Meridian. By J. Ivory, Esq. M.A. F.R.S.*

A FTER so many laborious researches undertaken to deter$A$ mine the figure of the earth, the opinions of philosophers upon that point are still very unsettled. In proof of this it will be sufficient to cite what is advanced in the latest memoir on this subject, in which it is said that the compression $\frac{1}{3} \overline{0}$. is adopted, because it is the mean between $\frac{1}{3 \frac{1}{1} 0}$ and $\frac{1}{2} \frac{1}{8}$, the limits between which the ellipticity is generally supposed to be comprised $t$. There is at least great prudence in this procedure; for at the same time that the particular ellipticity is pitched upon which is nearly the best fitted to reconcile all the phænomena with the measurements of the Survey, there is no risk incurred that the truth, when it can no longer be disputed, will fall beyond the boundaries mentioned. In the Numbers of this Journal for May and June last, the elliptical elements of the earth are deduced from the five most esteemed measurements of meridional arcs in our possession ; and it is fully proved that the elements found represent the five distances on the meridian with great accuracy. As far as our present knowledge extends, we are therefore entitled to infer that the terrestrial meridians are equal ellipses of a known excentricity, and the earth itself an elliptical spheroid having a known compression at the poles.

Till additional surveys shall enable us to establish the foregoing conclusion, or to correct it, if it be erroneous, we may inquire what light will be thrown on the question by measurements perpendicular to the meridian. In this Journal for July last, I have shown that, in the English Survey, the operations at Beachy Head and Dunnose, for finding the length of a degree perpendicular to the meridian, lead to a result that accords exactly with the same spheroid deduced from the meridional arcs. My present intention is to examine some more instances of the same kind; and I shall begin with putting the formula I used for computing the difference of longitude into a form more convenient for calculation.

* Communicated by the Author. † Phil. Trans. 1828, p. 132. 
Referring to this Journal, July last, pp. 8 and 9, for the explanation of the symbols employed, I shall now put

$$
\begin{aligned}
& \operatorname{Sin} \frac{\beta}{2}=\frac{\gamma}{2 a} \\
& \operatorname{Sin} \frac{\delta}{2}=\frac{1}{2} \sqrt{(p-u)^{2}+(q-t)^{2}}:
\end{aligned}
$$

and it is obvious that $\sin \frac{\delta}{2}$ is no other than half the chord of the elliptical meridian comprehended between the latitudes of the two stations, which may be computed to any required degree of exactness. If now we substitute these values in the equation at the top of p. 9 , we shall get,

$$
\operatorname{Sin}^{2} \frac{\beta}{2}-\sin ^{2} \frac{\delta}{2}=p u \sin ^{2} \frac{\alpha}{2} ;
$$

and hence,

$\operatorname{Sin} \frac{\omega}{2}=\sqrt{\frac{\sin \frac{\beta+\delta}{2} \sin \frac{\beta-\delta}{2}}{\cos \lambda \cos \lambda^{\prime}}} \times\left(1-\frac{8}{2} \sin ^{2} \lambda-\frac{8^{\prime}}{2} \sin ^{2} \lambda^{\prime}\right)$. or, in logarithms,

$$
\begin{aligned}
& \log \sin \frac{\omega}{2}=\frac{1}{2} \log \left(\frac{\sin \frac{\beta+\delta}{2} \sin \frac{\beta-\delta}{2}}{\cos \lambda \cos \lambda^{\prime}}\right)-\frac{M_{\ell}}{2} \times \\
& \left(\sin ^{2} \lambda+\sin ^{2} \lambda^{\prime}\right) \text {. }
\end{aligned}
$$

This formula, when $\varepsilon=0$, coincides with the usual rule for

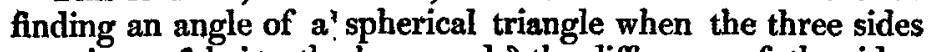
are given, $\beta$ being the base, and $\delta$ the difference of the sides. If we observe that small arcs of the elliptical meridian and of the equator, which are equal in length, have very nearly equal chords, we shall readily obtain this formula for finding $\delta$ which is very convenient in practice, viz.

$$
\delta=\left(\lambda-\lambda^{\prime}\right) \cdot\left\{1-\varepsilon\left(\frac{1}{2}+\frac{3}{2} \cos \left(\lambda+\lambda^{\prime}\right)\right)\right\}
$$

I shall now add another formula for finding the difference of longitude when there is given, the azimuth at one station, and the latitude of the other, together with the length of the chord between them. Let $m$ denote the azimuth at the first station, that is, the angle between the meridian and the second station; $\lambda^{\prime}$, the latitude of the second station ; and $\gamma$, the length of the chord: further put $\mathbf{R}$ for the radius of a sphere the surface of which passes through the two stations, and touches the horizon of the first: then the difference of longitude $w$, 
will be found by this formula, which is exact and easily demonstrated by the most simple geometry, viz.

$$
\operatorname{Sin} \omega=\frac{\gamma}{a} \cdot \frac{\sin m}{\cos \lambda^{\prime}} \sqrt{1-\frac{\gamma^{2}}{4 R^{2}}} \times \sqrt{1-2 \varepsilon \sin ^{8} \lambda^{\prime}} .
$$

Now it is obvious that $R$ will always be very nearly equal to $a$; and since $\gamma$ is always a small part of $\mathrm{R}$, or of $a$, we may take $\frac{\gamma^{2}}{4 a^{2}}$ as equivalent to $\frac{\gamma^{2}}{4 \mathrm{R}^{2}}$. But if we make $\sin \frac{\beta}{2}=\frac{\gamma}{2 a}$; then $\cos \frac{\beta}{2}=\sqrt{1-\frac{\gamma^{2}}{4 a^{2}}} ;$ and $\frac{\gamma}{a} \sqrt{1-\frac{\gamma^{2}}{4 a^{2}}}=2 \sin \frac{\beta}{2}$ $\cos \frac{\beta}{2}=\sin \beta:$ and thus we obtain,

or, in lograrithms,

$$
\operatorname{Sin} \omega=\frac{\sin \beta \sin m}{\cos \lambda^{\prime}} \sqrt{1-2 \varepsilon \sin ^{2} \lambda^{\prime}}
$$

$$
\log \sin \omega=\log \left(\frac{\sin \beta \sin m}{\cos \overline{\lambda^{\prime}}}\right)-M E \sin ^{2} \lambda^{\prime} .
$$

In illustration of these rules I am tempted to apply them for finding the difference of longitude between the observatories at Greenwich and Paris. In the new survey the length of the arc drawn from Dover perpendicular to the meridian of Greenwich, is 50634 fathoms*. I consider the foot of this arc as the first station in the formula (B), and Dover as the second station. Hence, $m=90^{\circ} ; \lambda^{\prime}=50^{\circ} 7^{\prime} 45^{\prime \prime} \cdot 6$. As the given distance is not a chord, but an arc on the earth's surface, we shall find $\beta$ by reducing the given length, taken as an arc of the earth's equator, to degrees : therefore $\beta=\frac{50634}{60856} \times 3600^{\prime \prime}$ $=49^{\prime} 53^{\prime \prime} \cdot 3$. The formula (B) will now give us the longitude of Dover equal to

$$
1^{\circ} 19^{f} 23^{\prime \prime} \cdot 78 \text {. }
$$

As we have no azimuth either at Dover or Dunkirk, we must apply the formula (A). The two latitudes are,

Dover............ $\lambda=51^{\circ} 7^{\prime} 45^{\prime \prime} \cdot 6$

Dunkirk......... $\lambda^{\prime}=51 \quad 2 \quad 8 \cdot 5$.

General Roy makes the distance from Dover to Dunkirk equal to 244916 feet $\dagger$, or 40822 imperial fathoms. According to the mode of calculation in the Survey, this length is not a chord, but an arc on the earth's surface; and hence $\beta=\frac{40822}{60856} \times 3600^{\prime \prime}$ $=40^{\prime}$ 14".87. The formula (a) gives $\delta=5^{\prime} 36^{\prime \prime} \cdot 91$. We have therefore, by the formula $\left(A^{\prime}\right)$, the difference of longitude between Dover and Dunkirk equal to

$$
1^{\circ} 3^{\prime} 19^{\prime \prime} \cdot 10 \text {. }
$$

* Phil. Trans. 1828, p. 180.

+ Trig. Survey, vol. i. p. 147 . 
The sum of the two results is the longitude of Dunkirk, $2^{\circ} 22^{\prime} 42^{\prime \prime} \cdot 88$ : and as the meridian of Paris is $2^{\prime} 22^{\prime \prime}$ west of the meridian of Dunkirk*, we get the difference of longitude of the two observations, equal to,

$$
\begin{aligned}
& \text { In degrees. } \\
& 2^{\circ} 20^{\prime} 20^{\prime \prime} \cdot 88 \quad \ldots . . . . . . . . . . . . . .99^{m} 21^{\text {s.39 }} \\
& \text { By experiment, P. T. 1826, } 921 \cdot 46 \\
& \text { Defect... } 0.07
\end{aligned}
$$

In order to confirm the result obtained at Beachy Head, I shall add a similar instance taken from the New Survey. At the station of Fairlight $f$, the angle between the meridian and Blancnez, was found $85^{\circ} 36^{\prime} 36^{\prime \prime} \cdot 73=m$; the latitude of Blancnez is $50^{\circ} 55^{\prime} 29^{\prime \prime} \cdot 36=\lambda^{\prime}$. The arc on the earth's surface between the two stations is 42117.6 fathoms; and hence $\beta=\frac{42117 \cdot 6}{60856} \times 3600^{\prime \prime}=41^{\prime} 31^{\prime \prime} .51$. The formula (B) will now give us the difference of longitude of the two stations equal to $1^{\circ} 5^{\prime} 33^{\prime \prime} \cdot 42$. According to the Survey, the length of the perpendicular arc at Blancnez is $4.1998^{\circ} 66$ fathoms; and the amplitude of this arc, computed from the difference of longitude, is $41^{\prime} 19^{\prime \prime} \cdot 58$; and hence, by proportion, the perpendicular degree is 60976 fathoms. Now, at the latitude of Blancnez, a perpendicular degree on the surface of the spheroid is $60974 \cdot 5$ fathoms.

It appears that the perpendicular degrees at Beachy Head and at Blancnez on the French coast, agree very exactly with the elliptical spheroid deduced from the meridional arcs. The coincidence of the curvature of the earth's surface with the same spheroid in this region, may likewise be inferred from the calculation of the difference of longitude between Greenwich and Paris, which brings out a result so near the quantity obtained by direct observation. Let us next inquire how the case will stand at a very distant part of the globe. Colonel Lambton has made in India a measurement precisely similar to that at Beachy Head and Dunnose in the British Survey. The particulars of this measurement at the two stations are as follows $\ddagger$ : Curnatighur.

Azimuth, $92^{\circ} 49^{\prime} 15^{\prime \prime} \cdot 93=m$, Azimuth, $87^{\circ} \quad 0^{\prime} \quad 7^{\prime \prime} \cdot 54$

Latitude, $1234 \quad 38 \cdot 85, \quad$ Latitude, $123212 \cdot 27=\lambda$ !

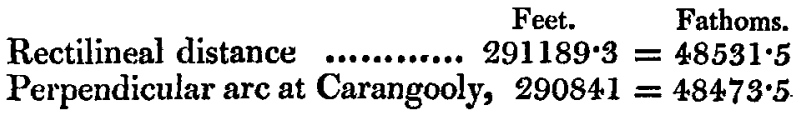

* Conn.des Tems. † Phil. Trans. p. 186.

$\ddagger$ Asiatic Researches, vol, viii. 
As in this instance we have the rectilineal distance of the stations, or the chord $\gamma$ between them, we must find $\beta$ by the formula $\sin \frac{\theta}{2}=\frac{\gamma}{2 a}=\frac{\gamma}{2 p \Delta}$; whence $\beta=47^{\prime} 50^{\prime \prime} \cdot 94$. With this value of $\beta$, and the values of $m$ and $\lambda^{\prime}$ noted above, the difference of longitude will be found, by the formula $(B)$, equal to $48^{\prime} 57^{\prime \prime} \cdot 05$. From this we get $47^{\prime} 47^{\prime \prime} \cdot 03$, for the amplitude of the perpendicular arc at Carangooly, and 60866 fathoms, for the perpendicular degree, very little different from $60865^{\circ} 2$, the length on the surface of the spheroid at the latitude of Carangooly.

But if the measurements made in England and India are all represented by the same spheroid, Why should not the case be the same in France? We have a great tendency to infer uniformity.in the works of Nature, which principle is in reality the foundation of every physical inquiry. And if the public were put in possession of the extensive operations that have been executed in France and the north of Italy, for determining an are of the mean parallel, there can be little doubt that we should be able to prove that all the degrees of the parallel are equal, and agree in their length with the dimensions of the spheroid we have been considering. But at present we cannot draw our arguments. from so rich a source, and we shall be content with examining a single instance taken from the great meridional measurement of France.

The length of an arc drawn perpendicular to the meridian of Dunkirk from La Rogière, in latitude $44^{\circ} 34^{\prime} 36^{\prime \prime} \cdot 6$ is, according to the survey, 27534.6 toises *. This length is a deduction from actual measurement, and is independent of any hypothesis about the figure of the earth. The arc is $29345 \cdot 2$ fathoms; and, by proceeding as in the example of Dover, we get $\beta=28^{\prime} 55^{\prime \prime} \cdot 95$, and the difference of longitude $=40^{\prime} 33^{\prime \prime} \cdot 21$. In order to find the amplitude of the arc, we must know the latitude of the point where it cuts the meridian. Now, according to the survey, the small arc of the meridian, between the foot of the perpendicular and the parallel of La Rogière, is $=114.3$ toises $=121.7$ fathoms, making $7^{1 / .2}$ of difference of latitude. The latitude of the foot of the perpendicular is therefore, $44^{\circ} 34^{\prime} 43^{\prime \prime} \cdot 8$; whence we get the amplitude $=$ $28^{\prime} 53 ! \cdot 18$, and the length of the perpendicular degree $=$ 60953 fathoms, exactly the same as on the surface of the spheroid at the latitude $44^{\circ} 34^{\prime} 43^{\prime \prime} \cdot 8$.

In this last instance, as well as in all the others, the degree perpendicular to the meridian measured on the earth's surface,

* Base Metrique, vol. iii. p. 268.

Nero Series. Vol. 4. No.21. Sept. 1828. 
is very consistent with the dimensions of the spheroid deduced from the meridional arcs. Now La Rogière is only $1^{\circ} 9^{\prime}$ south of the parallel on which the measurements have been made in France and Italy; and it is well known that the result of these operations requires $n$ compression considerably different from what we have investigated. Here there is a difficulty of some moment, which it would be interesting to discuss, but which the length of this article precludes us from entering upon at present.

August $8,1828$.

J. Ivory.

XXXIV. The Tables, of Oltmanns for calculating Heights by the Barometer, rendered applicable to English Barometers and Measures. By H. T. DE LA BEche, Esq.F.R.S.*

THE French Board of Longitude have given these Tables in their Annuaire for the last two or three years, and state that they appear to them "the most convenient of all those hitherto published, for facilitating the calculation of heights." After this eulogium it would be useless for me to add any thing in favour of their merits, the chief of which consists in their great simplicity.

Being calculated for the metrical barometer, these tables were useless to persons employing that graduated according to English inches and their decimal parts. To render them applicable to our barometers, I have prefixed a table $(A)$, in which the equivalent of every millimetre of the metrical barometer is given in English inches and the hundredth parts of inches, which is sufficiently close for all practical purposes.

To reduce the metres used in these tables into English feet, I have appended a table (F), where the number of English feet corresponding to any number of metres up to 10,000 will be immediately abtained.

Abstraction being made of table A prefixed, and table $\mathrm{F}$ appended, the march of operations is as follows:

Let $h$ be the height of the barometer at the lower station expressed in millimetres; $h^{\prime}$ that of the higher station; T and $T^{\prime}$ the temperature of the barometer at the different stations sccording to the centigrade thermometer, $t$ and $t^{t}$ that of the air.

We search in table $B$ for the number which corresponds to $h$; let us call it $a$ : we likewise search in the same table for that which corresponds to $h^{\prime}$; let this be named $b$ : let us call

- Communicated by Mr. De la Beche. 